\title{
High Variability of Whole-Blood Tacrolimus Pharmacokinetics Early After Thoracic Organ Transplantation
}

\author{
Maaike A. Sikma ${ }^{1}$ - Claudine C. Hunault ${ }^{2}$ - Erik M. Van Maarseveen ${ }^{3,4}$. Alwin D. R. Huitema ${ }^{4,5}$.

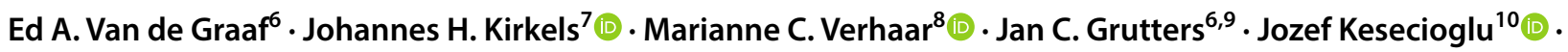 \\ Dylan W. De Lange ${ }^{11}$ (D)
}

Published online: 19 November 2019

(c) The Author(s) 2019

\begin{abstract}
Background and Objective Oral tacrolimus is initiated perioperatively in heart and lung transplantation patients. There have been few studies on oral tacrolimus pharmacokinetics early post-transplantation, even though tacrolimus-related toxicity may occur early, potentially leading to morbidity and mortality. Therefore, we aimed to study the pharmacokinetics of oral tacrolimus in thoracic organ recipients during the first days after transplantation.

Methods We conducted a pharmacokinetic study in 30 thoracic organ transplants at intensive care at the University Medical Center Utrecht in the first week post-transplantation. Twelve-hour whole-blood tacrolimus profiles were examined using high-performance liquid chromatography tandem mass spectrometry (HPLC-MS/MS) and analysed via population pharmacokinetic modelling.

Results The concentration-time profiles showed high variability. Concentrations at $12 \mathrm{~h}$ were outside the target range in $69 \%$ of the cases. A two-compartment model with mixed first-order and zero-order absorption adequately described tacrolimus concentrations. The typical value of the apparent clearance was $19.6 \mathrm{~L} / \mathrm{h}$ (95\% CI 16.2-22.9), and the apparent distribution volumes of central and peripheral compartments, V1 and V2, were 231 L (95\% CI 199-267) and 521 L (95\% CI 441-634), respectively. Inter-occasion (dose-to-dose) variability far exceeded the interindividual variability (IIV), with an estimated variability in relative bioavailability of 55\% (95\% CI 48.5-64.4).

Conclusions The high variability of tacrolimus pharmacokinetics early after thoracic organ transplantation is largely due to excessive variability in bioavailability, making individualised dosing based on measured concentrations futile. To bypass this bioavailability issue, we suggest administering tacrolimus intravenously and aiming below the upper therapeutic range early post-transplantation.

Clinical Trial Registraion: NTR 3912/EudraCT 2012-001909-24.
\end{abstract}

\section{Introduction}

The immunosuppressant tacrolimus, which is a calcineurin inhibitor, is used extensively in thoracic organ transplantation patients. Tacrolimus is generally administered orally

The authors confirm that the PI for this paper is M.A. Sikma and that she had direct clinical responsibility for patients.

Electronic supplementary material The online version of this article (https://doi.org/10.1007/s13318-019-00591-7) contains supplementary material, which is available to authorized users.

Maaike A. Sikma

m.a.sikma@umcutrecht.nl

Extended author information available on the last page of the article because of the suspected hepatotoxicity and nephrotoxicity of the solvent polyoxyl 60 hydrogenated castor oil (HCO60 ) used in intravenous formulations [1, 2]. Unfortunately, tacrolimus has a narrow therapeutic range, making it difficult to attain therapeutic targets in clinically unstable patients, such as patients early after heart and lung transplantation $[3,4]$. Yet, adequate therapeutic exposure is very important, because a variable tacrolimus concentration increases the risk of transplanted organ dysfunction and death [5-7]. A supratherapeutic whole-blood tacrolimus trough concentration in the first week after thoracic transplantation has been related to acute kidney injury (AKI), which is, on its own, a risk factor for poor outcome $[3,4,8-10]$. Therefore, pharmacokinetic-guided dosing is of vital importance and is now commonplace. The most important prerequisite for 


\section{Key Points}

In the first week after thoracic organ transplantation, the inter-occasion (dose-to-dose) variability of pharmacokinetic parameters were shown to be far higher than the interindividual variability (IIV), and were found to be mainly due to excessive variability in bioavailability.

Such huge variabilities hamper any attempt to predict the appropriate tacrolimus concentration for the next dose based upon previous concentrations measured during the first days post-transplantation.

Theoretically, tacrolimus therapy may be optimised in clinically unstable patients by circumventing the bioavailability issue through the use of intravenous administration.

appropriate dosing based on measured drug concentrations is that exposure after dose adaptation can be adequately predicted based on the measured exposure after previous doses. This requires that the dose-to-dose variability is relatively low compared to the interindividual variability (IIV). As a consequence, knowledge of the complex pharmacokinetics of tacrolimus in clinically unstable thoracic organ recipients is crucial. However, only a few studies on the pharmacokinetics of oral tacrolimus early after thoracic organ transplantation have been performed. Those studies showed that the pharmacokinetics varied with the patient group considered; for instance, a $40 \%$ lower bioavailability was observed in cystic fibrosis (CF) patients, and a $40 \%$ higher clearance in cytochrome P450 3A5 (CYP3A5) expressers [11-17]. Also, a low clearance rate has been observed in heart transplant recipients early post-transplantation [18]. The interoccasion (dose-to-dose) variability (IOV) of oral tacrolimus pharmacokinetics early post-transplantation was studied by Miano et al. Their study showed that CYP3A5 combined with CYP3A4*22 expression along with clinical factors such as the use of CYP450 inhibitors (azole antifungals, amiodarone), the transplant type, a diagnosis of cystic fibrosis and haematocrit accounted for $42 \%$ of the variance in the dose-corrected concentration during the first 2 weeks after lung transplantation [19]. Organ dysfunction (such as gut dysmotility and liver dysfunction) and the use of extended extracorporeal life support were not taken into account. Thoracic organ recipients often experience prolonged surgery times, ischaemia of the transplanted organ(s), reperfusion oedema, acute rejection, bleeding and massive blood transfusions or infection, which result in shock, inflammation and organ failure $[20,21]$. Due to all of the abovementioned changes, altered drug bioavailability, distribution, metabolism and clearance will influence the pharmacokinetics of tacrolimus early after thoracic organ transplantation.
We hypothesised that thoracic organ recipients show high variability in 12-h post-dose whole-blood concentrations of tacrolimus ( $\mathrm{C} 12 \mathrm{~h}$ ) during the first days after transplantation. We analysed oral tacrolimus pharmacokinetics in 10 heart and 20 lung transplants within the first 6 days after transplantation.

\section{Patients and Methods}

\subsection{Compliance with Ethical Standards}

This was a descriptive and prospective study in 10 heart and 20 lung transplantation patients in the first 6 days after transplantation. Informed consent was obtained from all individual participants included in the study. The accredited review board for human studies of the University Medical Center Utrecht (UMC Utrecht) approved the study (NTR 3912/EudraCT 2012-001909-24).

\subsection{Patients}

All consecutive thoracic organ recipients who were admitted to the intensive care unit (ICU) of the UMC Utrecht between June 2013 and March 2015 and met the inclusion criteria but not the exclusion criteria were studied. The inclusion criteria were patients older than 18 years who were treated with tacrolimus and provided informed consent. Exclusion criteria were patients who died within 1 day after admission, had known allergies to tacrolimus and macrolides, or received total parenteral nutrition.

\subsection{The Immunosuppressive Regimen}

Tacrolimus (Prograf ${ }^{\circledR}$, Astellas Pharma Europe) was dosed orally twice daily (bid). The initial dose was $0.1 \mathrm{mg} / \mathrm{kg}$ bid for the lung transplantation patients and $2 \mathrm{mg}$ bid for the heart transplantation patients, and was started the day after transplantation. Dose adjustments were based on wholeblood tacrolimus concentrations $12 \mathrm{~h}$ post-dose $(\mathrm{C} 12 \mathrm{~h})$ taking drug-drug interactions, gut dysmotility and liver injury into account, and were left at the discretion of the attending physicians. The therapeutic range was from 9 to $15 \mathrm{ng} / \mathrm{ml}$ for all patients [22]. Steady state had not necessarily been reached when dose adjustments were made, in accordance with common clinical practice.

The immunosuppressive regimen of heart recipients further consisted of corticosteroids [prednisolone $50 \mathrm{mg}$ intravenously directly postoperative, followed by $25 \mathrm{mg}$ bid] and mycophenolate mofetil (MMF) [1000 mg orally bid]. For lung transplantation recipients, the immunosuppressive regimen consisted of basiliximab induction therapy on postoperative days 1 and 4 [20 $\mathrm{mg}$ per day intravenously], 
corticosteroids [prednisolone $25 \mathrm{mg}$ four times per day (qid) intravenously, tapered to $30 \mathrm{mg}$ once daily orally after 4 days] and MMF [starting dose $1500 \mathrm{mg}$ orally bid, tapered to $1000 \mathrm{mg}$ bid].

\subsection{Tacrolimus Analyses}

Twelve-hour whole-blood tacrolimus concentrations were determined daily from the transplantation date until 6 days after transplantation as long as the patients were admitted to the intensive care. Blood samples were collected daily during one dose interval [sampled pre-dose and at $1,1 \frac{1}{2}$, $2,21 / 2,3,4,6,8$, and $12 \mathrm{~h}$ after administration]. The stability of tacrolimus was tested for up to 1 week at room temperature. There was no decline in the tacrolimus concentration in whole-blood samples. All whole-blood samples were analysed within $24 \mathrm{~h}$. Analysis of tacrolimus was performed using high-performance liquid chromatography tandem mass spectrometry (HPLC-MS/MS) (Thermo Fisher Scientific), with a lower limit of quantification of $0.5 \mathrm{ng} / \mathrm{ml}$ and an intraday imprecision of $<5 \%$. The HPLC-MS/MS method was adapted from and validated according to the latest European Medicines Agency (EMA) guidelines [23]. The on-column stability was $24 \mathrm{~h}$ and the assay runtime was a maximum of $8 \mathrm{~h}$. Assay specificity was $100 \%$ due to the use of HPLC-MS/MS. Cross-reactivity with drugs commonly used in thoracic organ transplant recipients was tested for, and no interference was detected. The assay had a linear dynamic range of $1-50 \mathrm{ng} / \mathrm{ml}$. The between-run and between-day imprecisions (as measured by coefficients of variation) were within $10 \%$, and the bias was under $3 \%$. Low, median and high controls were all within $15 \%$. Furthermore, 5 years of results from an international interlaboratory proficiency testing program for tacrolimus showed that measurements for all external quality controls were within $15 \%$ of each other.

\subsection{Covariates}

Severity of illness was gauged using the Sequential Organ Failure Assessment (SOFA) score, which was recorded in the intensive care unit once a day (see also Table S1 in the Electronic supplementary material, ESM, for a description of the covariates). Shock was determined as a mean arterial pressure of $\leq 60 \mathrm{mmHg}$ or the infusion of at least one inotropic or vasopressive agent. Systemic inflammatory response syndrome (SIRS) was defined according to the definition of the American College of Chest Physicians (ACCP) and the Society of Critical Care Medicine Consensus Conference (SCCM) [24]. Diarrhoea and ileus were documented, as well as liver injury. Daily fluid balance and body weight were recorded. Drugs influencing the pharmacokinetics of tacrolimus were recorded, including drugs that potentially increase the blood concentration of tacrolimus by inhibiting or through substrate competition for CYP3A4/5 enzymes and the ATP-binding cassette subfamily B member 1 (ABCB1) transporter, as well as drugs that potentially decrease the blood concentration of tacrolimus by inducing CYP3A4/5 enzymes or the ABCB1 transporter [20, 25].

\subsection{Nephrotoxicity}

Renal function was evaluated during the first 6 days as long as the patient was admitted to the intensive care, and at 1 month after transplantation in the outpatient department (see also Table S1 in the ESM). Renal clearance was measured by analysing the creatinine concentration in 24-h urine. The occurrence of AKI and augmented renal clearance (ARC) as well as the use of continuous venovenous hemofiltration $(\mathrm{CVVH})$ were monitored.

\subsection{Population Pharmacokinetic Analysis}

The nonlinear mixed effects modelling software tool NONMEM (version 7.3.0) was used to model tacrolimus pharmacokinetics. The program Piraña (version 2.9.4) was used as an interface for NONMEM, and R for Windows (version 3.3.1) was employed to analyse the results.

The area under the concentration-time curve (AUC, in $\mathrm{ng} \cdot \mathrm{h} / \mathrm{mL}$ ) was computed using the following equation (Eq. 1):

$\mathrm{AUC}=\mathrm{F} \times \mathrm{AMT} / \mathrm{CL}$

where $\mathrm{F}$ is the relative bioavailability, AMT is the amount of drug (in $\mu \mathrm{g}$ ) and CL is the clearance (in $\mathrm{L} / \mathrm{h}$ ).

\subsection{Population Pharmacokinetic Modelling}

A two-compartment linear model with first-order oral absorption was used. On some occasions, the first observation within 30 min after dosing was the maximum concentration in the dose interval, indicating extremely rapid absorption. For those occasions, to reduce the complexity of the absorption model, dosing was treated as zero-order oral absorption with the duration of absorption equal to the time interval between dosing and the first observation (Fig. 1). The structural model included the following parameters: CL/F (apparent clearance), Q/F (intercompartmental clearance), V1/F (volume of distribution of the central compartment), V2/F (volume of distribution of the peripheral compartment) and $k_{\mathrm{a}}$ (absorption rate constant). IIV and IOV were described assuming a log normal distribution with the following equation (Eq. 2):

$P_{k j m}=\theta_{k} \times \mathrm{e}^{\left(\eta_{k j}+\kappa_{k m}\right)}$ 


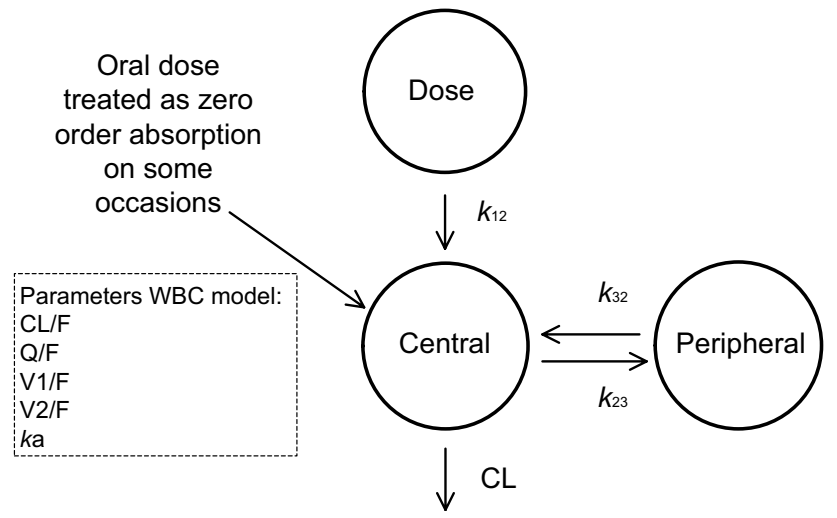

Fig. 1 Schematic representation of the population pharmacokinetic whole-blood concentration (WBC) model for tacrolimus. The absorption phase is described by a rate constant $\left(k_{12}\right)$. The oral dose was treated as IV administration on some occasions. The central compartment of volume $\mathrm{V}_{1} / \mathrm{F}$ is in rapid equilibrium with the peripheral compartment of volume $V_{2} / F$. Drug transfer between this peripheral compartment and the central compartment is described with the intercompartmental clearance parameter $\mathrm{Q} / \mathrm{F}$ using the following equations: $k_{23}=\mathrm{Q} / \mathrm{V}_{1}$ and $k_{32}=\mathrm{Q} / \mathrm{V}_{2}$. The volumes of distribution for tacrolimus can be estimated from this model. CL/F is the clearance of tacrolimus

where $P_{k j m}$ is the estimate for parameter $k$ for the $j$ th individual at occasion $m, \theta_{k}$ is the population value for the $k$ th pharmacokinetic parameter, $\eta_{k j}$ represents the IIV, which is assumed to have a normal distribution with a mean of 0 and a standard deviation of $\omega k$, and $\kappa_{k m}$ represents the IOV, which is assumed to have a mean of 0 and a standard deviation of $\pi k$. Although the absolute bioavailability was not identifiable, the variability in the relative bioavailability was estimated similarly with $\theta_{k}$ fixed at 1 .

The residual error was assumed to be proportional to the predicted concentration (Eq. 3):

$C_{i j}=\operatorname{Cpred}_{i j}\left(1+e_{i j}\right)$

where $C_{i j}$ is the $i$ th observation for the $j$ th individual, Cpred $_{i j}$ is the tacrolimus concentration predicted by the model, and $e_{i j}$ is the difference between $C_{i j}$ and Cpred $_{i j}$.

The modelling process was performed using the stochastic approximation expectation maximisation (SAEM) estimation method with interaction. The likelihood was subsequently established using the Monte Carlo importance sampling EM assisted by mode a posteriori (IMPMAP) estimation method. Parameter precision was estimated using the SIR (sampling importance resampling) procedure [26]. Concentrations below the lower limit of quantification (LLOQ) were discarded (3.9\%; 46 values out of 1180). Model evaluation was performed by several statistical (e.g. objective function, parameter precision) and graphical (goodness-offit plot) methods.

\subsection{Statistical Analyses}

Variables are presented below as the median (with the first and third quartiles $(\mathrm{Q} 1 ; \mathrm{Q} 3)$ ), range or number (proportion) where appropriate.

\section{Results}

\subsection{Patient Characteristics}

Thirty patients were included (see Table 1 for their characteristics, as well as Tables S1 and S2 in the ESM for more details and for definitions of the covariates). No patient died within the timeframe of the study. No organ rejection was observed in a 1-year time period. Twenty-six patients were supported with a cardiopulmonary bypass during surgery. Furthermore, 9 patients (7 lung transplants) were supported with extended extracorporeal membrane oxygenation (ECMO) for a median duration of 4 days $(\mathrm{Q} 1 ; \mathrm{Q} 32 ; 6)$. The median SOFA score was $7(\mathrm{Q} 1 ; \mathrm{Q} 34 ; 12)$. The frequency of shock was 93\% (28 out of 30) and that of SIRS was 100\% (30 out of 30). Gut dysmotility was observed in $97 \%$ of the patients ( 29 out of 30 ), with ileus in $90 \%$ of the patients ( 27 out of 30 ) and diarrhoea in $60 \%$ of the patients (18 out of 30 ), respectively. Three of 10 cystic fibrosis patients showed diarrhoea from days 2 and 3 on. All CF patients had exocrine pancreatic insufficiency. Pancreas enzymes were substitued when enteral feeding was implemented. Potential drug-drug interactions were observed in all patients. The number of drugs that potentially increased tacrolimus concentrations ranged from 0 to 6 . The number of drugs that potentially decreased tacrolimus concentrations ranged from 0 to 2 . Median baseline creatinine was $66 \mu \mathrm{mol} / \mathrm{L}(\mathrm{Q} 1 ; \mathrm{Q} 3$ 53;98). Median baseline creatinine clearance was $85 \mathrm{ml} / \mathrm{min} 1.73 \mathrm{~m}^{2}$ (Q1;Q3 73;116). Augmented renal clearance was seen in 7 out of 30 patients $(23 \%)$, with a median duration of 1 day (Q1;Q3 1;2). AKI was observed in $47 \%$ of the patients $(14$ out of 30). The frequency of patients with AKI who showed recovery of creatinine clearance at 1 month was $64 \%$ (9 out of 14).

\subsection{Pharmacokinetic Profiles}

The total number of whole-blood $\mathrm{C} 12 \mathrm{~h}$ profiles was 119 , with a median of 5 profiles per patient (range 1-6). Characteristic features of the whole-blood tacrolimus concentrations together with the dosages are illustrated in Fig. 2.

\subsection{Observed Pharmacokinetics}

The median $\mathrm{C} 12 \mathrm{~h}$ was $9.5 \mathrm{ng} / \mathrm{ml}$ (range 0.5-38.7). Most of the whole-blood concentrations, $69.4 \%$, were outside the 
Table 1 Patient characteristics $(\mathrm{n}=30)$

\begin{tabular}{|c|c|c|}
\hline Variable & $N(\%)$ & Median (Q1; Q3) \\
\hline Male & $15(50 \%)$ & - \\
\hline Age (year) & - & $43(34 ; 60)$ \\
\hline Bodyweight (kg) & & $73.5(61 ; 86)$ \\
\hline Length $(\mathrm{cm})$ & & $173.5(169 ; 176)$ \\
\hline \multicolumn{3}{|l|}{ Reason for transplantation } \\
\hline \multicolumn{3}{|l|}{ Heart $(N=10)$} \\
\hline Ischaemic CMP & $5(16.7 \%)$ & - \\
\hline Non-ischaemic CMP & $5(16.7 \%)$ & - \\
\hline \multicolumn{3}{|l|}{ Lung $(N=20)$} \\
\hline Cystic fibrosis & $10(33.3 \%)$ & - \\
\hline COPD & $4(13.3 \%)$ & - \\
\hline ILD & $6(20 \%)$ & - \\
\hline Double lung transplantation & $18(90 \%)$ & - \\
\hline \multicolumn{3}{|l|}{ Parameters } \\
\hline SOFA score & & $7(4 ; 12)$ \\
\hline SIRS at least once between days 1 and 6 & $30(100 \%)$ & \\
\hline SIRS duration (days) & & $4.5(3 ; 6)$ \\
\hline Shock at least once between days 1 and 6 & $28(93.3 \%)$ & \\
\hline Shock duration (days) & & $2(1 ; 3)$ \\
\hline Liver dysfunction at least once between days 1 and 6 & $14(47 \%)$ & \\
\hline Gut dysmotility frequency & $29(96.7 \%)$ & \\
\hline Ileus at least once between days 1 and 6 & $27(90 \%)$ & \\
\hline Ileus duration & & $2(2 ; 3)$ \\
\hline Diarrhea at least once between days 1 and 6 & $18(60 \%)$ & \\
\hline Diarrhea duration & & $1(0 ; 2)$ \\
\hline \multicolumn{3}{|l|}{ Fluid balance (L/day) } \\
\hline Day 1 & & $1.5(0.2 ; 3)$ \\
\hline Day 2 & & $1.2(0.4 ; 2.2)$ \\
\hline Day 3 & & $0.5(-0.2 ; 1.5)$ \\
\hline Day 4 & & $0.4(-0.8 ; 0.9)$ \\
\hline Day 5 & & $-0.3(-1.0 ; 1.1)$ \\
\hline Day 6 & & $-0.2(-1.4 ; 0.0)$ \\
\hline \multicolumn{3}{|l|}{ Change in bodyweight from baseline (kg) } \\
\hline Day 1 & & $1.5(0 ; 6)$ \\
\hline Day 2 & & $6.5(0.8 ; 11)$ \\
\hline Day 3 & & $8(0 ; 14)$ \\
\hline Day 4 & & $10(0.5 ; 14.5)$ \\
\hline Day 5 & & $7(-1 ; 14.5)$ \\
\hline Day 6 & & $5(-0.8 ; 18.5)$ \\
\hline Postoperative ECMO frequency & $8(27 \%)$ & \\
\hline Postoperative ECMO duration (days) & & $4(2 ; 6)$ \\
\hline \multicolumn{3}{|l|}{ Tacrolimus } \\
\hline Tacrolimus $\mathrm{C} 12 \mathrm{~h}$ (ng/ml) (min-max) & & $9.5(0.5-38.7)$ \\
\hline$C_{\max }(\mathrm{ng} / \mathrm{ml})$ & & $18.5(2.1-74.7)$ \\
\hline$T_{\max }(\mathrm{h})$ & & $1.6(0.4-8.0)$ \\
\hline AUC (ng.h/mL) & & $151.2(31.2-2525)$ \\
\hline$T_{1 / 2}(\mathrm{~h})$ & & $9.4(6.0-31.4)$ \\
\hline Patients with at least one drug that increased tacrolimus levels between days 1 and 6 & $30(100 \%)$ & \\
\hline Number of drugs that increased the tacrolimus concentration (min-max) & & $0-6$ \\
\hline Patients with at least one drug that decreased tacrolimus levels between days 1 and 6 & $30(100 \%)$ & \\
\hline
\end{tabular}


Table 1 (continued)

\begin{tabular}{|c|c|c|}
\hline Variable & $N(\%)$ & Median (Q1; Q3) \\
\hline Number of drugs that decreased the tacrolimus concentration (min-max) & & $0-2$ \\
\hline \multicolumn{3}{|l|}{ Renal function } \\
\hline Baseline creatinine $(\mu \mathrm{mol} / \mathrm{L})$ & & $66(53 ; 98)$ \\
\hline Baseline creatinine clearance $\left(\mathrm{ml} / \mathrm{min} / 1.73 \mathrm{~m}^{2}\right)$ & & $85(73 ; 116)$ \\
\hline $\mathrm{ARC}$ at least once between days 1 and 6 & $7(23.3 \%)$ & \\
\hline ARC duration (days) (min-max) & & $1(1-6)$ \\
\hline AKI at least once between days 1 and 6 & $14(47 \%)$ & \\
\hline AKI recovery at 1 month & $9^{\mathrm{a}}(64 \%)$ & \\
\hline
\end{tabular}

SOFA sequential organ failure assessment, SIRS systemic inflammatory response syndrome, ECMO extracorporeal membrane oxygenator, $C 12 \mathrm{~h}$ concentration $12 \mathrm{~h}$ after administration, $C_{\max }$ maximum $\mathrm{C} 12 \mathrm{~h}, T_{\max }$ time to maximum concentration, $A U C$ area under the concentration-time curve, $T_{I / 2}$ terminal half-life, $A R C$ augmented renal clearance, $A K I$ acute kidney injury, $C M P$ cardiomyopathy, $C O P D$ chronic obstructive pulmonary disease, $I L D$ interstitial lung disease

${ }^{a}$ Of 14 patients

target range (9-15 $\mathrm{ng} / \mathrm{ml})$. Subtherapeutic concentrations were observed in $51 \%$ and supratherapeutic concentrations were observed in $19 \%$ of the patients. The median of the maximum concentration $\left(C_{\max }\right)$ was $18.5 \mathrm{ng} / \mathrm{ml}$ (range 2.1-74.7). The median of the time to maximum concentration $\left(T_{\max }\right)$ was $1.6 \mathrm{~h}$ (range $\left.0.4-8\right)$.

\subsection{Pharmacokinetic Model for Tacrolimus}

A two-compartment model with first-order absorption or zero-order absorption in a few cases best fitted the data (for a schematic illustration of the pharmacokinetic model, see Fig. 1). The parameter precision was acceptable for all relevant parameters, as indicated by the low $95 \%$ confidence intervals (CIs) for the parameter estimates, indicating that all of the parameters could be reliably estimated (see Table 2). Goodness-of-fit plots of whole-blood tacrolimus concentrations (Fig. 3) showed that the data were widely dispersed around the unity line when the model with the population parameter estimates (upper right panel) was applied.

The IOV in relative bioavailability was estimated at $55.0 \%$ (95\% CI 48.5-64.4). The IOV in apparent clearance was $29.5 \%$ (95\% CI 20.7-38.9), and the IOV in the apparent distribution volume was $35.1 \%$ (95\% CI 27.0-48.0). Only the IIV of CL/F could be estimated; the estimation of all other IIV elements yielded estimates close to 0 and/ or unsuccessful runs, most likely due to the fact that variability was dominated by the corresponding IOV. No further formal covariate analysis was conducted. Time-independent covariates (such as genotype or gender) are by definition not predictive for the observed IOV. The time-dependent covariates available (such as gut dysmotility, shock and corticosteroid use) changed much more slowly over time than the observed IOV. Furthermore, the magnitude of the change in each of these covariates was much lower than the IOV. Therefore, these covariates were unable to explain the extreme IOV observed. The median AUC was $151.2 \mathrm{ng} \cdot \mathrm{h} /$ $\mathrm{mL}$ (range 31.2-2525). The median terminal $T_{1 / 2}$ was $9.4 \mathrm{~h}$ (range 6.0-31.4).

\section{Discussion}

In this pharmacokinetic analysis involving 30 thoracic organ recipients, we showed that the bioavailability of tacrolimus displays a large IOV in the first week after transplantation. Other pharmacokinetic parameters also showed large IOVs. These high variabilities hamper attempts to predict appropriate next-dose tacrolimus concentrations based upon previous concentrations. In other words, pharmacokinetic-guided dosing seems to be of limited added value in clinically unstable patients. Nevertheless, daily therapeutic drug monitoring remains worthwhile for the prevention of toxicity, since subsequent doses will be omitted in the presence of high concentrations. In order to optimise tacrolimus therapy, it may be useful to circumvent bioavailability issues by administering it intravenously during clinical instability.

In general, tacrolimus is orally dosed after thoracic organ transplantation even when gut dysmotility ensues, because tacrolimus has known nephrotoxicity and intravenous administration may cause additional nephrotoxicity due to the use of the solvent HCO-60 [1, 2]. When the oral route is not feasible, sublingual administration has been recommended over intravenous administration by some authors, though this is not sufficiently substantiated yet and is therefore not common practice [27].

Very few studies have analysed the pharmacokinetics of oral tacrolimus in clinically unstable transplantation patients $[17,18,28]$. Unfortunately, none of these studies reported severity of illness, shock or organ failure, hampering any comparison of the outcomes. Our cohort of thoracic organ transplant patients showed high SOFA scores, and many had 

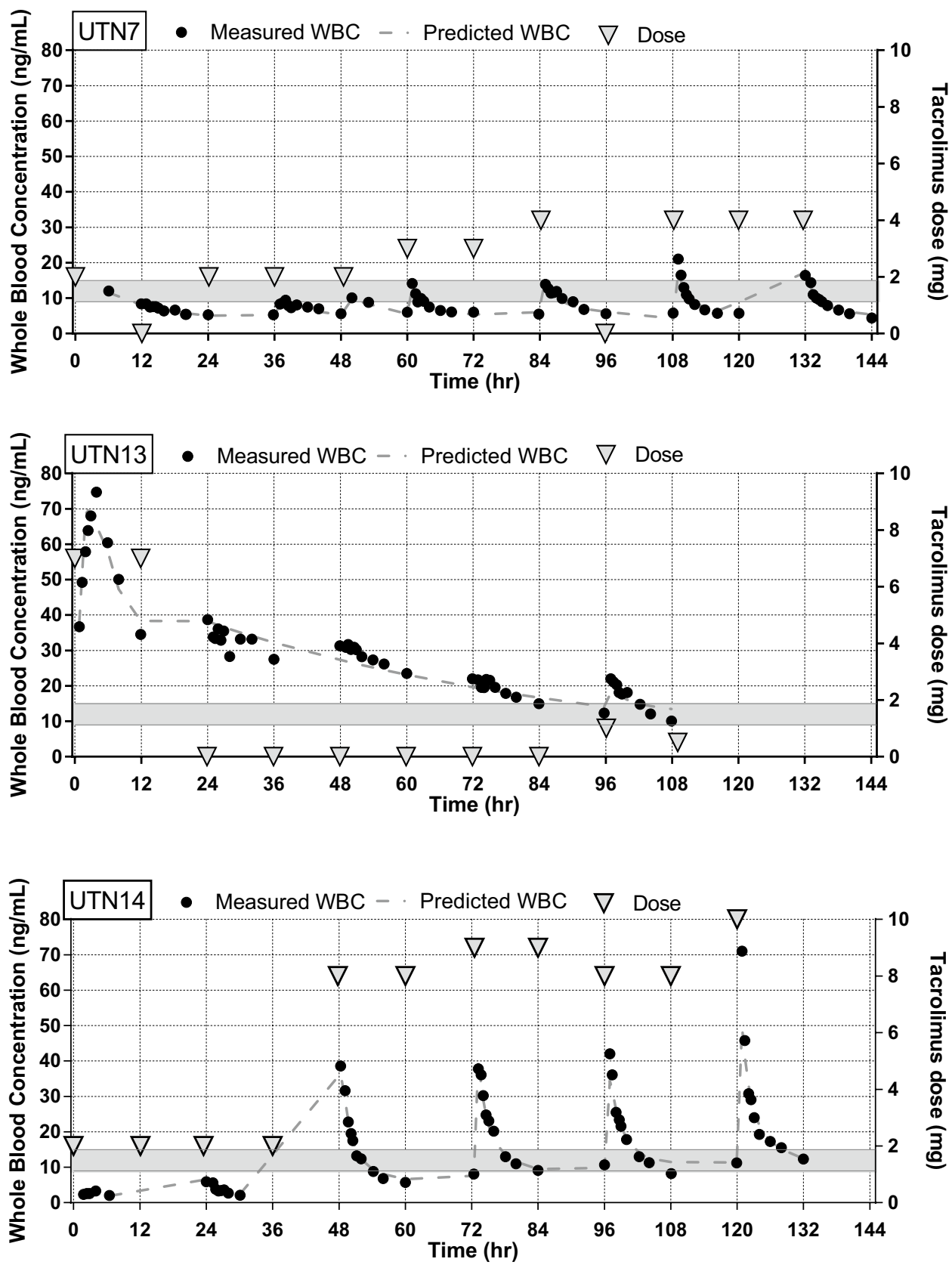

Fig. 2 Three illustrative individual whole-blood concentration (WBC) profiles showing the administered tacrolimus dose and the individual predicted lines. The therapeutic range for tacrolimus $\mathrm{C} 12 \mathrm{~h}$ is indicated by grey bars. UTN7: This patient presented $\mathrm{C} 12 \mathrm{~h}$ values below the therapeutic range, despite increasing the dosage. The patient was a heart transplant recipient with shock and circulatory support for 4 days. Gut dysmotility was observed for 3 days. UTN13: In this patient, absorption was rapid and complete, with a short $T_{\max }$ and a high $C_{\max }$. This patient was an uncomplicated non-CF lung transplantation patient. Cardiopulmonary bypass was used during surgery. Hyperdynamic circulation in combination with augmented renal clearance existed for 3 days. Diarrhoea was observed on days
4 and 5. UTN14: This patient was a heart transplant recipient and had severe bleeding during surgery, for which two red blood cell units were administered on day 1 . For 5 days, he was supported with ECMO, vasopressors and inotropes because he was in shock. Fluid balance ranged from 1 to 4 litres. No gut dysmotility occurred. Very low $\mathrm{C} 12 \mathrm{~h}$ values were measured during days 1 and 2 , which would support the idea of low absorption or distribution into the red blood cells. High clearance was observed from day 3 to day 6 . High doses were needed to achieve a therapeutic $\mathrm{C} 12 \mathrm{~h}$. $C F$ cystic fibrosis, $C 12$ concentration at $12 \mathrm{~h}, C_{\max }$ maximum $\mathrm{C} 12$ concentration, $T_{\max }$ time to reach $\mathrm{C}_{\max }$ 
Table 2 Final population pharmacokinetic parameters with 95\% confidence intervals, as obtained using SIR

\begin{tabular}{llll}
\hline $\begin{array}{l}\text { Pharma- } \\
\text { cokinetic } \\
\text { parameter }\end{array}$ & $\begin{array}{l}\text { Estimate }(95 \% \\
\text { CI) }\end{array}$ & $\begin{array}{l}\text { Interpatient vari- } \\
\text { ability } \%(95 \% \\
\text { CI) }\end{array}$ & $\begin{array}{l}\text { Inter-occasion } \\
\text { variability } \% \\
(95 \% ~ C I)\end{array}$ \\
\hline $\mathrm{CL} / \mathrm{F}(\mathrm{L} / \mathrm{h})$ & $19.6(16.2-22.9)$ & $34.6(24.2-48.6)$ & $29.5(20.7-38.9)$ \\
$\mathrm{V}_{1} / \mathrm{F}(\mathrm{L})$ & $231(199-267)$ & n.e. & $35.1(27.0-48.0)$ \\
$k_{\mathrm{a}}(1 / \mathrm{h})$ & $0.579(0.456-$ & n.e. & $98.3(81.1-121)$ \\
& $0.778)$ & & \\
$\mathrm{Q} / \mathrm{F}(\mathrm{L} / \mathrm{h})$ & $58.2(49.7-69.3)$ & n.e. & n.e. \\
$\mathrm{V}_{2} / \mathrm{F}(\mathrm{L})$ & $521(441-634)$ & n.e. & n.e. \\
$\mathrm{F}$ & Fixed at 1 & n.e. & $55.0(48.5-64.4)$ \\
Residual & $14.0(13.3-14.6)$ & & \\
$\quad$ unex- & & & \\
plained & & & \\
variability & & & \\
$(\%)$ & & & \\
\hline
\end{tabular}

SIR sampling importance resampling, n.e. not estimated, $C I$ confidence interval, $k_{a}$ absorption rate constant, $C L / F$ apparent clearance, $V 1 / F$ central compartment volume of distribution, $V 2 / F$ peripheral compartment volume of distribution, $Q / F$ intercompartmental clearance, $F$ bioavailability

shock in the first week, corresponding to major physiological instability. We observed a high inter-occasion (i.e. betweendose) variability in bioavailability independent of the dose, indicating unpredictable uptake of tacrolimus, which is probably related to this clinical instability. This contrasts with kidney transplant recipients early after transplantation, in whom bioavailability was observed to be dose dependent and had a much smaller IOV of only 23-28\% [29, 30]. Renal transplant patients are often clinically stable, and many do not need ICU support in the days after transplantation. Tacrolimus target concentrations are frequently reached in renal recipients within 3 days, which is different from our cohort of heart and lung recipients [31]. Despite the large differences in absorption variability, the observed clearance as well as the variability in clearance was in accordance with earlier reports $[14,16,28,30,32]$. The distribution volumes in our cohort were only slightly higher than those in another study conducted 1 week after lung transplantation, and both studies had similar distribution volume IOVs [14].

The high IOV observed for tacrolimus pharmacokinetics in our study population may be explained by a range of factors, all of which are associated with clinical instability. First, delayed absorption as well as very early absorption were observed in our data, which may be partly explained by a high incidence of gut dysmotility in all of our patients with and without $\mathrm{CF}$. Ileus delays tacrolimus transport to the duodenum, where it is mainly absorbed [33]. On the other hand, the prompt absorption of tacrolimus with a sudden peak in blood concentration may be due to a short transit time to the duodenum, which may be caused by a hyperdynamic state and (e.g. mycophenolate-associated) diarrhoea [33-35]. Furthermore, increased gut perfusion may increase absorption by augmented enteric drug extraction due to a large difference in intracellular and blood concentrations, and diarrhoea may increase absorption by shortening the transit time and maximising luminal degradation and dissolution of tacrolimus [36]. Moreover, diarrhoea, shock and inflammation may result in decreased ABCB 1 and CYP3A4/5 expression [37-39]. These phenomena could all increase tacrolimus transport and also shorten the transit time across the gut wall into the blood compartment, giving rise to sudden higher peak concentrations [33].

Second, the distribution of tacrolimus may fluctuate due to changes in diffusion into erythrocytes and reduced binding to (lipo)proteins caused by red blood cell transfusion, bleeding, dilution caused by fluid resuscitation, capillary leakage and the use of an extracorporeal circuit [40]. Anaemia and hypoalbuminaemia were observed in all the patients (see Table S1 in the ESM), which may lead to higher unbound tacrolimus concentrations with lower whole-blood levels at equivalent doses [28, 41, 42]. The application of an extracorporeal circuit, such as a cardiopulmonary bypass, ECMO or continuous renal replacement therapy, may significantly alter the disposition of tacrolimus because of adsorption to the bypass equipment, acute haemodilution, hypoalbuminaemia and hypothermia [43, 44]. Third, drug-drug interactions influence tacrolimus bioavailability. We observed drug-drug interactions in all patients. For example, high-dose corticosteroids induce CYP3A enzymes and ABCB1. Therefore, tapering corticosteroids decreases the activities of both and may greatly increase tacrolimus bioavailability, especially in CYP3A5 nonexpressers [37, 45, 46]. Last, changes in the clearance of tacrolimus might contribute to fluctuations in whole-blood concentrations. Shock and inflammation strongly influence organ function and subsequently the metabolisation rate of the liver [25, 47, 48].

All of these variations in the covariates of tacrolimus pharmacokinetics give rise to high fluctuations in wholeblood tacrolimus concentrations and hence a persistently high IOV in the first week after thoracic organ transplantation [20]. The IOV in bioavailability far exceeded other sources of variability. No covariate relationships were identified, as no covariate (time dependent or time independent) was identified that could explain the large variability in bioavailability. Yet, fluctuations in tacrolimus concentrations are a known significant risk factor for rejection, toxicity, and outcome deterioration in heart and lung transplants [3, 4, 7-10].

\subsection{Future Perspectives}

Circumventing the high variability in bioavailability by administering tacrolimus intravenously may improve 


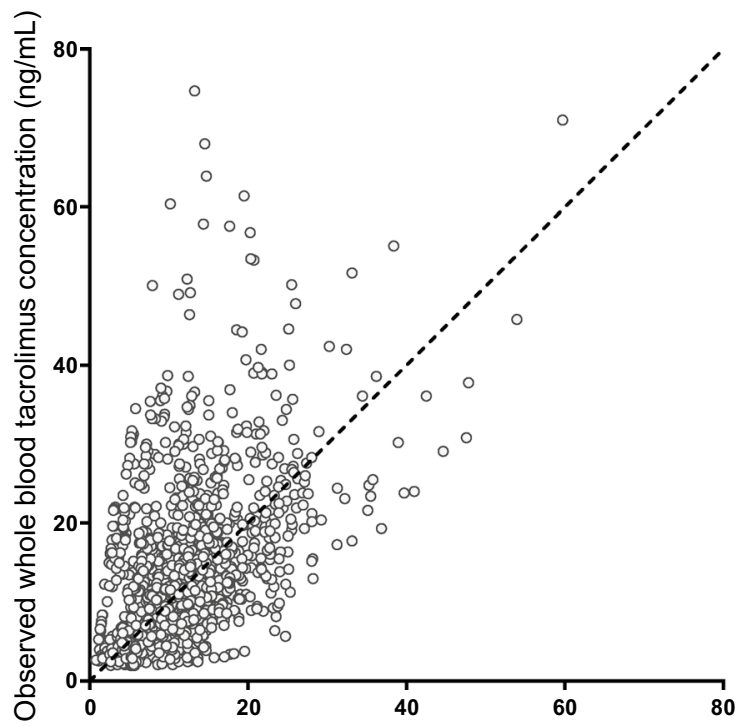

Model-predicted whole blood tacrolimus concentration $(\mathrm{ng} / \mathrm{mL})$

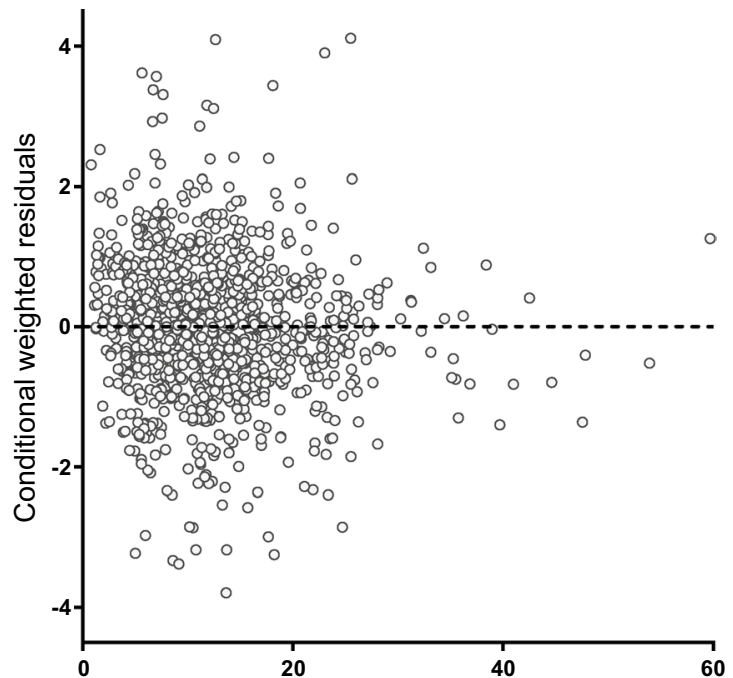

Population-predicted whole blood tacrolimus concentration $(\mathrm{ng} / \mathrm{mL})$

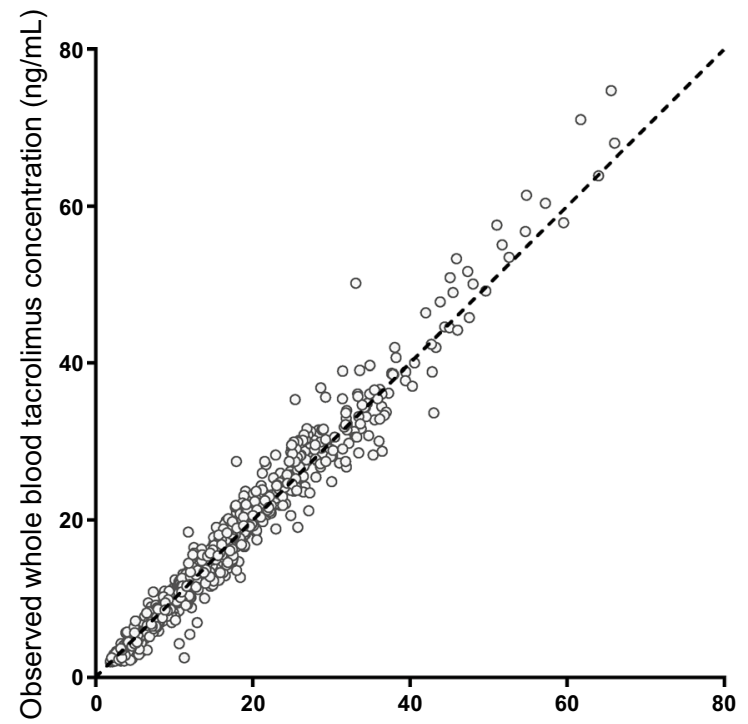

Individual-predicted whole blood tacrolimus concentration $(\mathrm{ng} / \mathrm{mL})$

Fig. 3 Goodness-of-fit plots

tacrolimus dosing despite the higher costs and the risk of additional nephrotoxicity from the solvent HCO-60 [2, 20, 49]. This will result in a decrease in tacrolimus concentration fluctuations and a subsequent decrease in the incidence of supratherapeutic $\mathrm{C} 12 \mathrm{~h}$. Therapeutic drug monitoring of $\mathrm{C} 12 \mathrm{~h}$ on a daily basis is mandatory in clinically unstable patients to improve dosing $[17,20]$. However, we showed that targeting the therapeutic range is impossible due to a high IOV. The therapeutic range of $10-15 \mathrm{ng} / \mathrm{ml}$, which is often used, is difficult to attain in clinically unstable patients [22]. Our findings support a more cautious approach. Targeting below the upper level of the therapeutic range (e.g. $15 \mathrm{ng} / \mathrm{ml}$ ) at day 5 after transplantation and not increasing the dose when low concentrations exist until clinical stability

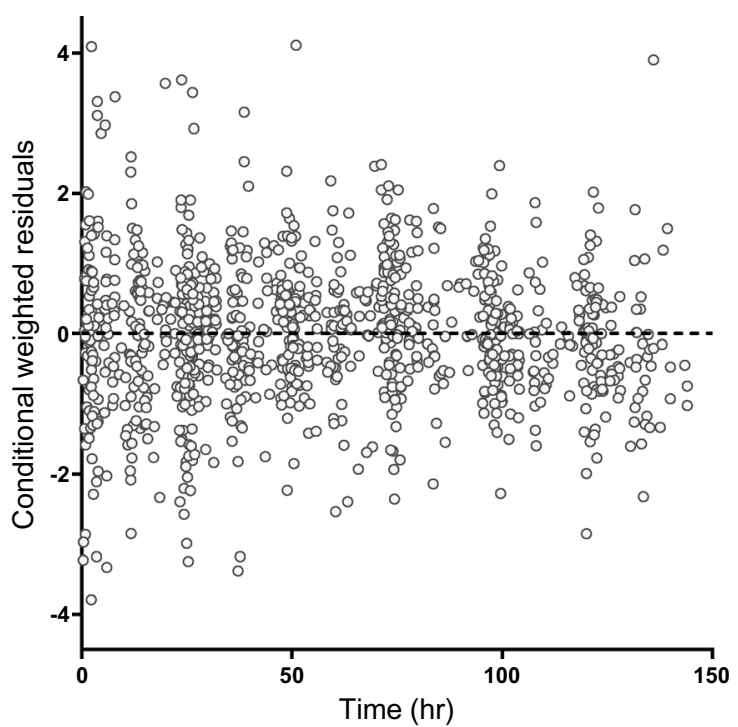

is established could help to prevent toxicity. Tacrolimus should be temporarily stopped when supratherapeutic concentrations arise. However, even lower therapeutic ranges might be advocated, as concentrations of $>10 \mathrm{ng} / \mathrm{ml}$ have already been associated with AKI [50]. It has even been suggested that an initial concentration of less than $4 \mathrm{ng} / \mathrm{ml}$ should be targeted, though early rejection may then arise, jeopardising the outcome [51]. To maintain adequate immunosuppression, corticosteroid dosage should not be tapered during clinical instability. In addition, accurate dosing of cell cycle inhibitors (e.g. MMF) and interleukin receptor blockers (e.g. basiliximab) is advocated to diminish the risk of rejection [11, 51-54]. In heart transplants, initiation of tacrolimus may even be postponed for 1 week when an 
interleukin receptor blocker is administered [55]. However, postponing tacrolimus may have very negative consequences for lung transplantation patients due to acute rejection [56].

\subsection{Study Limitations and Strengths}

Strong features of this study are the use of full 12-h pharmacokinetic profiles on a daily basis and the application of high-performance liquid chromatography with tandem mass spectrometry (HPLC-MS/MS) for analyses of blood concentrations. HPLC-MS/MS is a very accurate analytical method compared to immunoassays. However, some limitations of this study should also be noted. Overall, the sample size was relatively small, although these patients were sampled throughout during their ICU admission. Also, it is known that food impacts the absorption of tacrolimus [57], and enteral feeding was employed continuously in this study (it was not paused in any patient). We assume that the presence of ileus or diarrhoea will have had a major impact on absorption. The aim of this study was to examine tacrolimus pharmacokinetics in clinically unstable patients. These data are a good reflection of clinical practice, since large variations in whole-blood concentrations are frequently observed in the early phase after heart and lung transplantation.

\section{Conclusions}

Variability in whole-blood tacrolimus concentrations was excessive in clinically unstable thoracic organ transplantation patients. The pharmacokinetics of tacrolimus showed high IOVs. in particular, the IOV in relative bioavailability was excessive and was superimposed upon the variability in apparent distribution and apparent clearance. This makes pharmacokinetic-guided dosing to a preset narrow therapeutic range an impossible task in these patients. We suggest administering tacrolimus intravenously and aiming below the upper therapeutic range during the first few days after transplantation.

Author Contributions MAS, CCH, EMMVM, ADRH, EAVG, JHK, $\mathrm{MCV}, \mathrm{JCG}, \mathrm{JK}$, and DWDL wrote the article; MAS, CCH, EMM designed the research; MAS, CCH, EMM performed the research; MAS, CCH, EMM, ADRH, DWDL analysed the data.

\section{Compliance with Ethical Standards}

Funding No funding was received for this work.

Conflicts of interest Prof. Dr. Kesecioglu reports personal fees received from Xenios AG for an honorarium outside the submitted work. The other authors declare that there was no conflict of interest and no financial support. This research was presented as an abstract at the IATDMCT Congress 2018
Ethical approval All procedures performed in this study involving human participants were in accordance with the ethical standards of the institutional and/or national research committee and with the 1964 Helsinki Declaration and its later amendments or comparable ethical standards.

Informed consent Informed consent was obtained from all individual participants included in the study.

Open Access This article is distributed under the terms of the Creative Commons Attribution-NonCommercial 4.0 International License (http://creativecommons.org/licenses/by-nc/4.0/), which permits any noncommercial use, distribution, and reproduction in any medium, provided you give appropriate credit to the original author(s) and the source, provide a link to the Creative Commons license, and indicate if changes were made.

\section{References}

1. Collin C, Boussaud V, Lefeuvre S, Amrein C, Glouzman AS, Havard L, et al. Sublingual tacrolimus as an alternative to intravenous route in patients with thoracic transplant: a retrospective study. Transpl Proc. 2010;42:4331-7.

2. Worbs S, Köhler K, Pauly D, Avondet M-A, Schaer M, Dorner $\mathrm{MB}$, et al. Ricinus communis intoxications in human and veterinary medicine-a summary of real cases. Toxins (Basel). Mol Divers Preserv Int. 2011;3:1332-72.

3. Sikma MA, Hunault CC, van de Graaf EA, Verhaar MC, Kesecioglu J, de Lange DW, et al. High tacrolimus blood concentrations early after lung transplantation and the risk of kidney injury. Eur J Clin Pharmacol. 2017;73:573-80.

4. Sikma MA, Hunault CC, Kirkels JH, Verhaar MC, Kesecioglu J, de Lange DW. Association of whole blood tacrolimus concentrations with kidney injury in heart transplantation patients. Eur J Drug Metabol Pharmacokinet. 2018;43:311-20.

5. Gallagher HM, Sarwar G, Tse T, Sladden TM, Hii E, Yerkovich ST, et al. Erratic tacrolimus exposure, assessed using the standard deviation of trough blood levels, predicts chronic lung allograft dysfunction and survival. J Heart Lung Transplant. 2015;34:1442-8.

6. Rayar M, Tron C, Jézéquel C, Beaurepaire J-M, Petitcollin A, Houssel-Debry $\mathrm{P}$, et al. High intrapatient variability of tacrolimus exposure in the early period after liver transplantation is associated with poorer outcomes. Transplantation. 2018;102:e108-14.

7. Gueta I, Markovits N, Yarden-Bilavsky H, Raichlin E, Freimark $\mathrm{D}$, Lavee J, et al. High tacrolimus trough level variability is associated with rejections after heart transplant. Am J Transplant. 2018:18:2571-8.

8. Baran DA, Galin ID, Sandler D, Cheng J, Segura L, Courtney MC, et al. Predictors of early renal insufficiency in cardiac transplant recipients initiated on tacrolimus. TPS. 2002;34:1872-3.

9. Wehbe E, Duncan AE, Dar G, Budev M, Stephany B. Recovery from AKI and short- and long-term outcomes after lung transplatation. Clin J Am Soc Nephrol. 2013;8:19-25.

10. Tjahjono R, Connellan M, Granger E. Predictors of acute kidney injury in cardiac transplantation. Transpl Proc. 2016;48:167-72.

11. Baran DA, Galin ID, Zucker MJ, Alvi S, Arroyo LH, Lubitz S, et al. Can initial tacrolimus trough levels be predicted from clinical variables? Transpl Proc. 2004;36:2816-8.

12. Aidong W, Zhenjie C, Tong L, Lei Z, Yin W, Shanqi Z, et al. Therapeutic drug monitoring of tacrolimus in early stage after heart transplantation. TPS. 2004;36:2388-9. 
13. Saint-Marcoux F, Knoop C, Debord J, Thiry P, Rousseau A, Estenne M, et al. Pharmacokinetic study of tacrolimus in cystic fibrosis and non-cystic fibrosis lung transplant patients and design of Bayesian estimators using limited sampling strategies. Clin Pharmacokinet. 2005;44:1317-28.

14. Monchaud C, de Winter BC, Knoop C, Estenne M, ReynaudGaubert M, Pison C, et al. Population pharmacokinetic modelling and design of a Bayesian estimator for therapeutic drug monitoring of tacrolimus in lung transplantation. Clin Pharmacokinet. 2012;51:175-86.

15. Undre NA, Meiser BM, Uberfuhr P, Reichart B, Stevenson P, Schafer A, et al. Pharmacokinetics of tacrolimus (FK506) in primary orthoropic heart transplant patients. Transpl Proc. 1998;30:1112-5.

16. Uno T, Wada K, Matsuda S, Terada Y, Oita A, Kawase A, et al. Impact of the CYP3A $5 * 1$ allele on the pharmacokinetics of tacrolimus in Japanese heart transplant patients. Eur J Drug Metabol Pharmacokinet. 2018;43:665-73.

17. Molinaro M, Regazzi MB, Pasquino S, Rinaldi M, Iacona I, Campana $\mathrm{C}$, et al. Pharmacokinetics of tacrolimus during the early phase after heart transplantation. TPS. 2001;33:2386-9.

18. Undre NA, Stevenson PJ, European Tacrolimus Heart Study Group. Pharmacokinetics of tacrolimus in heart transplantation. TPS. 2002;34:1836-8.

19. Miano TA, Flesch JD, Feng R, Forker CM, Brown M, Oyster M, et al. Early tacrolimus concentrations after lung transplant are predicted by combined clinical and genetic factors and associated with acute kidney injury. Hoboken: Wiley; 2019.

20. Sikma MA, van Maarseveen EM, van de Graaf EA, Kirkels JH, Verhaar MC, Donker DW, et al. Pharmacokinetics and toxicity of tacrolimus early after heart and lung transplantation. Am J Transplant. 2015;15:2301-13.

21. Ascenzi P, Bocedi A, Notari S, Fanali G, Fesce R, Fasano M. Allosteric modulation of drug binding to human serum albumin. MRMC. 2006;6:483-9.

22. Brunet M, Van Gelder T, Åsberg A, Haufroid V, Hesselink DA, Langman L, et al. Therapeutic drug monitoring of tacrolimuspersonalized therapy: second consensus report. Ther Drug Monit. 2019;41:261-307.

23. Marinova M, Artusi C, Brugnolo L, Antonelli G, Zaninotto M, Plebani M. Immunosuppressant therapeutic drug monitoring by LC-MS/MS: workflow optimization through automated processing of whole blood samples. Clin Biochem. 2013;46:1723-7.

24. Dellinger RP, Levy MM, Rhodes A, Annane D, Gerlach H, Opal SM, et al. Surviving sepsis campaign: international guidelines for management of severe sepsis and septic shock: 2012. Crit Care Med. 2013;2013:580-637.

25. Christians U, Jacobsen W, Benet LZ, Lampen A. Mechanisms of clinically relevant drug interactions associated with tacrolimus. Clin Pharmacokinet. 2002;41:813-51.

26. Dosne A-G, Bergstrand M, Harling K, Karlsson MO. Improving the estimation of parameter uncertainty distributions in nonlinear mixed effects models using sampling importance resampling. $\mathrm{J}$ Pharmacokinet Pharmacodyn. 2016;43:583-96.

27. Doligalski CT, Liu EC, Sammons CM, Silverman A, Logan AT. Sublingual administration of tacrolimus: current trends and available evidence. Pharmacotherapy. 2014;34:1209-19.

28. Staatz C. Population pharmacokinetics of tacrolimus in adult kidney transplant recipients. Clin Pharmacol Ther. 2002;72:660-9.

29. Ekberg H, Mamelok RD, Pearson TC, Vincenti F, Tedesco-Silva $\mathrm{H}$, Daloze P. The challenge of achieving target drug concentrations in clinical trials: experience from the Symphony study. Transplantation. 2009;87:1360-6.

30. Størset E, Holford N, Hennig S, Bergmann TK, Bergan S, Bremer $\mathrm{S}$, et al. Improved prediction of tacrolimus concentrations early after kidney transplantation using theory-based pharmacokinetic modelling. Br J Clin Pharmacol. 2014;78:509-23.

31. Størset E, Åsberg A, Skauby M, Neely M, Bergan S, Bremer S, et al. Improved tacrolimus target concentration achievement using computerized dosing in renal transplant recipients-a prospective, randomized study. Transplantation. 2015;99(10):2158.

32. Han N, Ha S, Yun H-Y, Kim MG, Min S-I, Ha J, et al. Population pharmacokinetic-pharmacogenetic model of tacrolimus in the early period after kidney transplantation. Basic Clin Pharmacol Toxicol. 2014;114:400-6.

33. Sikma MA, van Maarseveen EM, Donker DW, Meulenbelt J. Letter to the editor: "Immunosuppressive drug therapy-biopharmaceutical challenges and remedies". Expert Opin Drug Deliv Inf Healthc. 2015;12:1955-7.

34. Hesselink DA, Bouamar R, Elens L, Van Schaik RHN, Van Gelder $\mathrm{T}$. The role of pharmacogenetics in the disposition of and response to tacrolimus in solid organ transplantation. Clin Pharmacokinet. 2014;53:123-39.

35. Tang JT, Andrews LM, Van Gelder T, Shi YY, van Schaik RHN, Wang LL, et al. Pharmacogenetic aspects of the use of tacrolimus in renal transplantation: recent developments and ethnic considerations. Expert Opin Drug Metab Toxicol. 2016;12:555-65.

36. Mittal N, Thompson JF, Kato T, Tzakis AG. Tacrolimus and diarrhea: pathogenesis of altered metabolism. Pediatr Transplant. 2001;5:75-9.

37. Kuypers DR, De Jonge H, Naesens M, Vanrenterghem Y. Effects of CYP3A5 and MDR1 single nucleotide polymorphisms on drug interactions between tacrolimus and fluconazole in renal allograft recipients. Pharmacogenet Genomics. 2008;18:861-8.

38. Lemahieu W, Maes B, Verbeke K, Rutgeerts P, Geboes K, Vanrenterghem Y. Cytochrome P450 3A4 and P-glycoprotein activity and assimilation of tacrolimus in transplant patients with persistent diarrhea. Am J Transplant. 2005;5:1383-91.

39. Nakamura A, Amada N, Haga I, Tokodai K, Kashiwadate T. Effects of elevated tacrolimus trough levels in association with infectious enteritis on graft function in renal transplant recipients. Transpl Proc. 2014;46:592-4.

40. Zahir H, Nand RA, Brown KF, Tattam BN. Validation of methods to study the distribution and protein binding of tacrolimus in human blood. J Pharmacol Toxicol Methods. 2001;46:27-35.

41. De Jonge H, Vanhove T, de Loor H, Verbeke K, Kuypers DRJ. Progressive decline in tacrolimus clearance after renal transplantation is partially explained by decreasing CYP3A4 activity and increasing haematocrit. Br J Clin Pharmacol. 2015;80:548-59.

42. Størset E, Holford N, Midtvedt K, Bremer S, Bergan S, Åsberg A. Importance of hematocrit for a tacrolimus target concentration strategy. Eur J Clin Pharmacol. 2014;70:65-77.

43. Shekar K, Roberts JA, Welch S, Buscher H, Rudham S, Burrows F, et al. ASAP ECMO: Antibiotic, Sedative and Analgesic Pharmacokinetics during Extracorporeal Membrane Oxygenation: a multi-centre study to optimise drug therapy during ECMO. BMC Anesthesiol. 2012;12:29.

44. Pea F, Pavan F, Furlanut M. Clinical relevance of pharmacokinetics and pharmacodynamics in cardiac critical care patients. Clin Pharmacokinet. 2008;47:449-62.

45. Christians U, Jacobsen W, Benet LZ. Mechanisms of clinically relevant drug interactions associated with tacrolimus. Clin Pharmacokinet. 2002;41:813-51.

46. Staatz CE, Størset E, Bergmann TK, Hennig S, Holford N. Tacrolimus pharmacokinetics after kidney transplantation-influence of changes in haematocrit and steroid dose. Br J Clin Pharmacol. 2015;80:1475-6.

47. Tata PNV, Subbotina N, Burckart GJ, Muddiman DC, Gusev AI, Hercules DM, et al. Species-dependent hepatic metabolism of immunosuppressive agent tacrolimus (FK-506). Xenobiotica. 2009;39:757-65. 
48. Lunde I, Bremer S, Midtvedt K, Mohebi B, Dahl M, Bergan S, et al. The influence of CYP3A, PPARA, and POR genetic variants on the pharmacokinetics of tacrolimus and cyclosporine in renal transplant recipients. Eur J Clin Pharmacol. 2014;70:685-93.

49. Snell GI, Ivulich S, Mitchell L, Westall GP, Levvey BJ. Evolution to twice daily bolus intravenous tacrolimus: optimizing efficacy and safety of calcineurin inhibitor delivery early post lung transplant. Ann Transplant. 2013;18:399-407.

50. Atalan HK, Gucyetmez B, Aslan S, Yazar S, Polat KY. Postoperative acute kidney injury in living donor liver transplantation recipients. Int J Artif Organs. 2017;41:37-42.

51. Knotek M, Mihovilović K, Galešić Ljubanović D, Maksimović B. Tacrolimus or mycophenolate in kidney transplantation--less, or more? Am J Transplant. 2014; 14:1220.

52. Zhang H, Fu Q, Zheng Y, Li J, Wang S, Deng R, et al. Effect of early immunosuppression therapy on de novo anti-human-leukocyte-antigen antibody after kidney transplantation. Transpl Proc. 2018;50:2382-7.

53. Zhang H, Liu L, Li J, Fu Q, Wan J, Deng R, et al. The efficacy and safety of intensified enteric-coated mycophenolate sodium with low exposure of calcineurin inhibitors in Chinese de novo kidney transplant recipients: a prospective study. Int J Clin Pract. 2016;70(Suppl 185):22-30.

54. Ekberg H, Bernasconi C, Tedesco-Silva H, Vítko S, Hugo C, Demirbas A, et al. Calcineurin inhibitor minimization in the Symphony study: observational results 3 years after transplantation. Am J Transplant. 2009;9:1876-85.

55. Kittipibul V, Tantrachoti P, Ongcharit $\mathrm{P}$, Ariyachaipanich A, Siwamogsatham S, Sritangsirikul S, et al. Low-dose basiliximab induction therapy in heart transplantation. Clin Transplant. 2017;31:e13132.

56. Yusen RD, Edwards LB, Dipchand AI, Goldfarb SB, Kucheryavaya AY, Levvey BJ, et al. The registry of the International Society for Heart and Lung Transplantation: thirty-third adult lung and heart-lung transplant report-2016; focus theme: primary diagnostic indications for transplant. J Heart Lung Transplant. 2016;35:1170-84.

57. Bekersky I, Dressler D, Mekki QA. Effect of low- and high-fat meals on tacrolimus absorption following $5 \mathrm{mg}$ single oral doses to healthy human subjects. J Clin Pharmacol. 2001;41:176-82.

\section{Affiliations}

\section{Maaike A. Sikma ${ }^{1}$ - Claudine C. Hunault ${ }^{2}$ - Erik M. Van Maarseveen ${ }^{3,4}$ - Alwin D. R. Huitema ${ }^{4,5}$ (D)

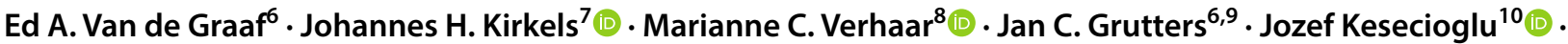 Dylan W. De Lange ${ }^{11}$}

1 Department of Intensive Care and Dutch Poisons Information Center, University Medical Center Utrecht, Utrecht University, F06.149, P.O. Box 85500, 3508 GA Utrecht, The Netherlands

2 Dutch Poisons Information Center, University Medical Center Utrecht, University Utrecht, Utrecht, The Netherlands

3 Department of Clinical Pharmacy, Princess Máxima Center, University Medical Center Utrecht, University Utrecht, Utrecht, The Netherlands

4 Department of Clinical Pharmacy, University Medical Center Utrecht, University Utrecht, Utrecht, The Netherlands

5 Department of Pharmacy and Pharmacology, Netherlands Cancer Institute, Amsterdam, The Netherlands

6 Department of Lung Transplantation, University Medical Center Utrecht, University Utrecht, Utrecht, The Netherlands
7 Department of Cardiology, Heart Transplantation, University Medical Center Utrecht, University Utrecht, Utrecht, The Netherlands

8 Department of Nephrology and Hypertension, University Medical Center Utrecht, University Utrecht, Utrecht, The Netherlands

9 Department of Pulmonology, St. Antonius Hospital, Nieuwegein, Utrecht, The Netherlands

10 Department of Intensive Care, University Medical Center Utrecht, University Utrecht, Utrecht, The Netherlands

11 Dutch Poisons Information Center and Department of Intensive Care, University Medical Center Utrecht, University Utrecht, Utrecht, The Netherlands 